ODONTOLOGÍA SANMARQUINA

ISSN: 1560-9111

\section{Microfiltración apical en dientes obturados empleando la técnica de condensación lateral, cono único y nueva técnica propuesta}

Apical microleakage in teeth filled using lateral condensation technique, single cone and new proposal technique

\section{Artículo ORIGINAL}

Helly Páucar-Gutiérrez', Luis Maldonado-Huamaní1, Piero Palomares-Bustamante ${ }^{1}$ Sheyla Cáceres-Monzón ${ }^{1}$ Doris Salcedo-Moncada ${ }^{2}$ Luis Mallqui-Herrada ${ }^{1}$

\section{Resumen}

Objetivos: Comparar el grado de microfiltración apical utilizando tres técnicas de obturación. Materiales y método: 36 piezas dentarias fueron preparadas con limas rotatorias Mtwo hasta $\mathrm{N}^{\circ}$ 40/.04 para luego ser seleccionadas aleatoriamente utilizando tres técnicas de obturación (G1: técnica de conden-sación lateral; G2: cono único; G3: nueva técnica propuesta). Posteriormente, las piezas dentarias se barnizaron exponiendo los $4 \mathrm{~mm}$ apicales, se sumergieron en tinta china y se sometieron al proceso de descalcificación siguiendo el protocolo de Roberston, y se midió la filtración a través del microscopio estereoscópico. Las comparaciones fueron realizadas con la prueba de Kruskal Wallis. Resultados: Las medias en los tres grupos fueron: G1=0,17 $\pm 0,21 \mathrm{~mm}, \mathrm{G} 2=$ $0,34 \pm 0,49 \mathrm{~mm}$ y G3 $=0,14 \pm 0,21 \mathrm{~mm}$. Siendo de menor medida el grupo que se obturó con la nueva técnica propuesta. Conclusiones: La nueva técnica propuesta presentó menor filtración apical, sin embargo no se encontraron diferencias estadísticamente significativas entre las tres técnicas de obturación.

Palabras clave: Microfiltración, Obturación del conducto radicular, Endodoncia (Fuente: DeCS BIREME).

\section{Abstract}

Objectives: Compare the degree of apical microleakage using three filling techniques: lateral condensa-tion, single cone and a new proposed technique. Materials and method: 36 teeth were prepared with rotary files Mtwo until No. $40 / .04$ and then be randomly selected using three obturation techniques (G1: lateral condensation technique, G2: single cone; G3: new proposed technique). Subsequently, the teeth are varnished exposing the apical $4 \mathrm{~mm}$, dipped in ink and those undergoing the decalcification process following the Protocol of Roberston and measured seepage through a stereoscopic microscope. Comparisons were made with the Kruskal Wallis test. Results: The new proposed technique had a lower rate of microfiltration comparing the lateral condensation technique and single cone. No statistically significant differences between the three obturation techniques. Conclusions: The apical microleakage was similar among the three obturation techniques. Keywords: Microleakage, Root canal obturation, Endodontics.
1. Escuela Académico Profesional de Odontología de la Universidad Nacional Mayor de San Marcos.

2. Departamento de Estomatología Rehabilitadora. Sección Endodoncia y Carielogía de la Facultad de Odontología de la Universidad Nacional Mayor de San Marcos.

Correspondencia :

Helly Paucar Gutierrez: marysol_13_14@hotmail.com Mz. D 21 Lt. 8 Mariscal Caceres S.J. L, 013019771 , +51952352898

Coautores:

Luis Maldonado: luisalbertomh1992@hotmail.com Piero Palomares: pieropb_29@hotmail.com Sheyla Cáceres: sharoni41@hotmail.com Doris Salcedo: endodoncista@hotmail.com Luis Mallqui: Irj164@yahoo.com

Fecha de recepción: 10/11/2015 Fecha de aceptación: 25/4/2016

\section{Introducción}

Uno de los objetivos de la terapia endodóntica es la obturación tridimensional del sistema de conductos radiculares, impidiendo la reinfección y el crecimiento de los microorganismos residuales dentro del conducto, y en consecuencia, genera un ambiente biológicamente adecuado para permitir la cicatrización de los tejidos. ${ }^{1-2}$

Se han desarrollado diversos materiales y técnicas para conformar la obturación de los conductos radiculares. El objetivo de la obturación es la obliteración del canal radicular lo más cerca posible del CDC, (union conducto-dentinacemento), por medio de materiales inertes, dimensionalmente estables y bien tolerado por los tejidos periapicales y que además permitan un sellado, hermético, tridimensional y permanente. $^{3-5}$ El sellado del conducto radicular se evalúa, con frecuencia, por la filtración de fluidos: parámetro usado a menudo para enaltecer o condenar los materiales y las técnicas de obturación. Una obturación endodóntica, bien compactada y adaptada a la perfección, debe producir el cierre total de la interfase pared dentinaria. La tendencia es revisar y comparar las técnicas de obturación, con la finalidad de elegir la mejor y la más simple, para que el procedimiento sea más rápido, menos complicado y más accesible al profesional y al propio paciente. La filtración se genera debido a que la gutapercha no está bien compactada, producto del uso de una técnica de obturación inadecuada o mal empleada. ${ }^{6-10}$

El objetivo del estudio es comparar el grado de microfiltración apical de dientes obturados utilizando tres técnicas de obturación: condensación lateral, cono único y nueva técnica propuesta.

() Los autores. Este artículo es publicado por la Revista Odontología Sanmarquina de la Facultad de Odontología, Universidad Nacional Mayor de San Marcos. Este es un artículo de acceso abierto, distribuido bajo los términos de la licencia Creative Commons Atribucion - No Comercia_Compartir Igual 4.0 Internacional. (http://creativecommons.org/licenses/by-nc-sa/4.0/) que permite el uso no comercial, distribución y reproducción en cualquier medio, siempre que la obra original sea debidamente citada. Para uso comercial, por favor póngase en contactto con revista.odontologia@gmail.com. 


\section{Materiales y método}

Se recolectaron 36 piezas dentarias unirradiculares recientemente extraídas, mantenidas en suero fisiológico. L uego, se irrigó por 5 minutos con hipoclorito de sodio para eliminar los restos orgánicos. Las muestras se dividieron aleatoriamente en tres grupos: Grupo $1(\mathrm{n}=12)$ Técnica de condensación lateral usando cono principal No 40 de conicidad 03, Grupo 2 ( $n=12)$ Técnica de cono único usando cono $\mathrm{N}^{\circ} 40 / .04$ Mtwo, Grupo 3 ( $n=12)$ Nueva técnica propuesta (cono único + condensación lateral) usando cono principal de conicidad $\mathrm{N}^{\circ} 40 / .04$ Mtwo y conos accesorios de conicidad 02 (Figura 1).

Se seccionó la corona de cada diente extraído mediante un disco de carburundum, con la finalidad de emplear solo las raíces. Para obtener la longitud de trabajo, se introdujo una lima $\mathrm{K}$ No 10 o 15 hasta visualizarla a través del foramen apical y a la medida se le restó $1 \mathrm{~mm}$ para obtener la longitud de trabajo. Posteriormente, se instrumentó manualmente con limas $\mathrm{K}$ hasta la No 20; y se realizó la instrumentación rotatoria con el sistema Mtwo (10/.04, 15/.05, 20/.06, 25/.06, 30/.05, 35/.04, 40/.04) empleando el motor VDW. SILVER. La irrigación entre lima y lima fue con hipoclorito de sodio al 5,25\% y al finalizar se empleó EDTA al $17 \%$ para eliminar el smear layer. Se verificó la permeabilidad del conducto con una lima K No 10 a través del foramen, y se procedió a la conometría mediante el uso de radiovisógrafo. Para la obturación se empleó cemento tipo Grossman (ENDOFILL-Dentsply), se sellaron los conductos de acuerdo a la técnica seleccionada para cada grupo, se compactó verticalmente y la cavidad fue sellada con policarboxilato de zinc (DURELON-3M ESPE).

Las piezas fueron almacenadas en condiciones de humedad relativa, envolviendo cada raíz en una gasa húmeda con suero fisiológico, incubados a $37^{\circ} \mathrm{C}$ por 5 días para asegurar el completo fraguado del cemento sellador, se barnizó con dos capas de esmalte transparente de uñas y cera parafina a excepción de los $4 \mathrm{~mm}$ apicales. Luego las muestras se sumergieron en tinta china (Pelikan) y se centrifugaron a $3000 \mathrm{rpm}$ por 5 minutos, posteriormente se mantuvieron en inmersión pasiva por 3 días. Se enjuagaron en agua corriente y se retiró tanto el esmalte de uñas y la cera parafina con una hoja de bisturí No 15. Los especímenes fueron diafanizados mediante la técnica de Robertson, primero se descalcificaron en ácido nítrico al 5\% por 2 días cambiando el líquido cada 24 horas, luego se deshidrataron en concentraciones crecientes de alcohol etílico $\left(60^{\circ}, 80^{\circ}\right.$ y $\left.96^{\circ}\right)$ cada 5 horas y se transparentaron utilizando salicilato de metilo por 72 horas.
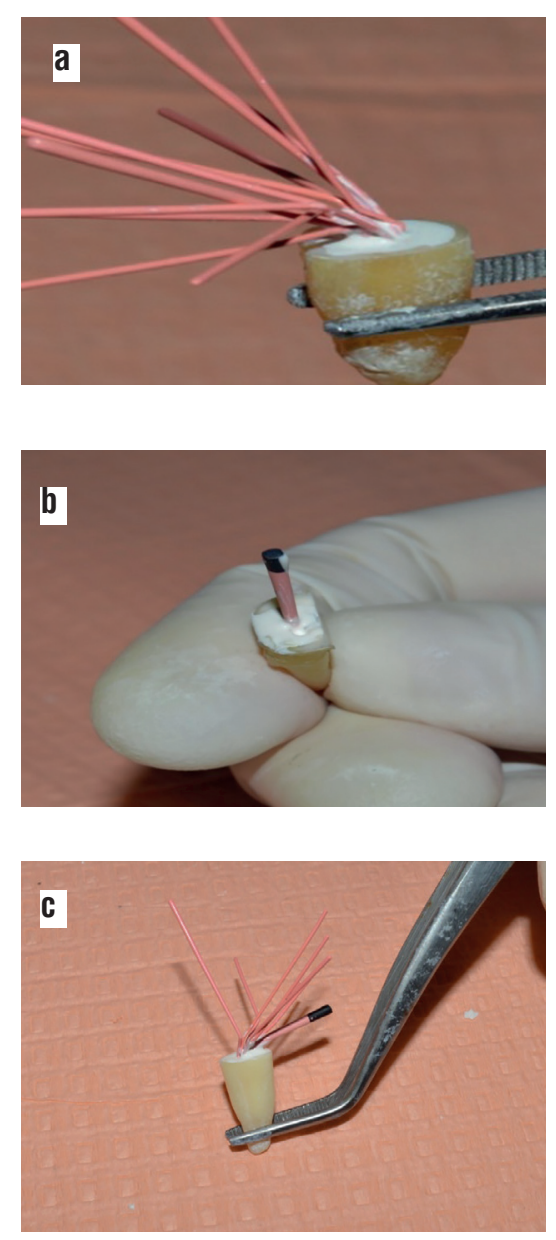

Fig. 1. Técnicas de obturación. a) Condensación lateral. b) Cono único. c) Nueva técnica propuesta.

Para evaluar la microfiltración, se observó al microscopio estereoscópico utilizando papel canson milimetrado y se tomaron fotografías. Utilizando el programa Image J, se calibró la distancia de $1 \mathrm{~mm}$, y finalmente se midió la filtración de cada diente desde el límite de la obturación hacia coronal (Figura 2). Los datos se procesaron en el programa para ciencias SPSS versión 21.0. En la prueba de normalidad (Shapiro-Wilk) se observó que existió dispersión, por lo que se utilizó la prueba no paramétrica de Kruskal Wallis, con un valor de significancia de 0,05.
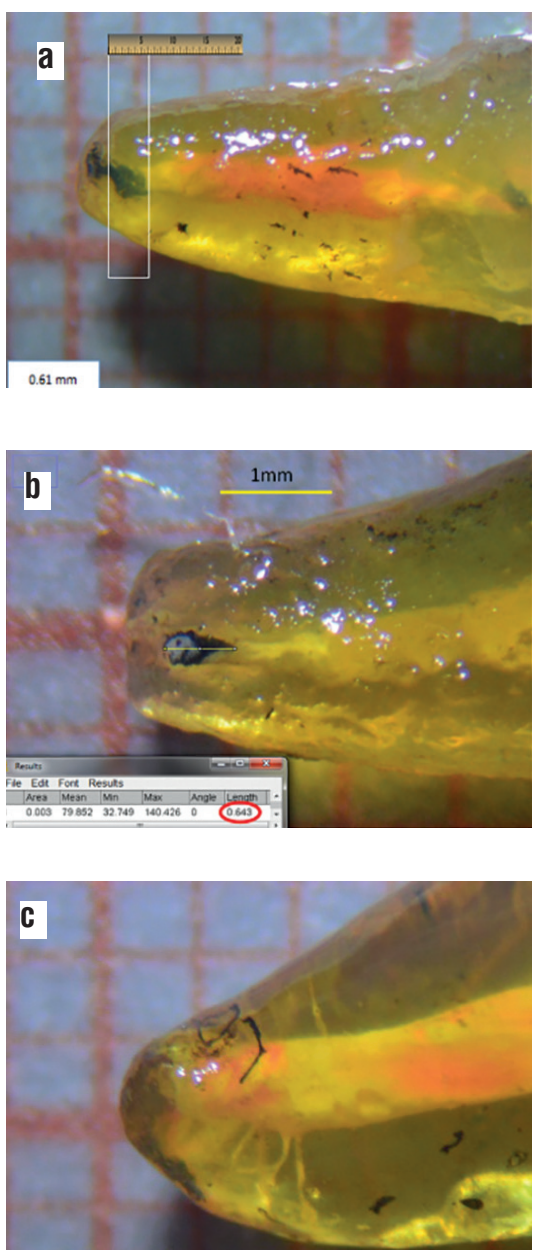

Fig. 2. Medida de filtración apical (mm) en microscópico estereoscópico mediante el programa Image J: a: condensación lateral: $0,61 \mathrm{~mm}$ b: cono único: 0,64 mm c: nueva técnica :0,0 $\mathrm{mm}$.

\section{Resultados}

Los resultados de la microfiltración apical de las 36 muestras se expresan en la Tabla 1, donde "n" representa el número de muestras utilizadas en cada grupo, la mediana (Me), media y desviación estándar (DE). Además se reportan el valor mínimo y máximo (rango) de filtración en milímetros para cada grupo.

En la tabla 1 se muestran las medias en los tres grupos: Grupo 1: Condensación lateral $=0,17 \pm 0,21 \mathrm{~mm}$, Grupo 2: Cono único $=0,34 \pm 0,49 \mathrm{~mm}$ $\mathrm{y}$ Grupo 3: Nueva técnica propuesta $=$ $0,14 \pm 0,21 \mathrm{~mm}$.

Según Kruskal Wallis, los valores no presentaron diferencias estadísticamente significativas entre los tres grupos $(\mathrm{p}=$ 0,567). 
Tabla 1. Microfiltración apical de tres técnicas de obturación.

\begin{tabular}{lccccccc}
\hline \multirow{2}{*}{ GRUPO } & \multicolumn{7}{c}{ Microfiltración apical } \\
& $\mathbf{n}$ & Media & DE & Me & Min & Max & $\mathbf{P}^{*}$ \\
\hline Cond. lateral & 12 & 0,17 & 0,21 & 0,05 & 0,00 & 0,61 & \\
Cono único & 12 & 0,34 & 0,49 & 0,06 & 0,00 & 1,54 & 0,57 \\
Nueva técnica & 12 & 0,13 & 0,21 & 0,00 & 0,00 & 0,54 & \\
\hline
\end{tabular}

*Prueba de Kruskal Wallis.

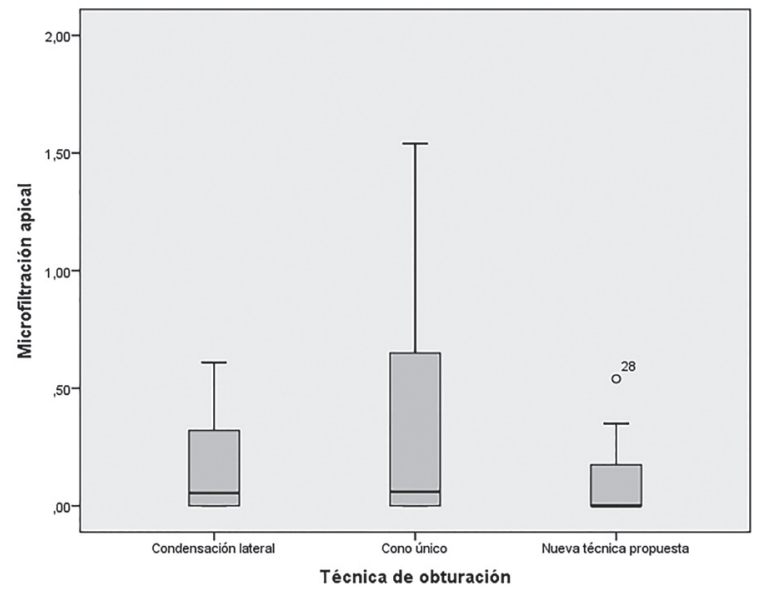

Gráfico 1. Diagrama de caja y bigotes.

Teniendo como referencia las medianas, se evidencia que la nueva técnica propuesta presenta cifras más cercanas al cero y tiene como valor máximo un $0,54 \mathrm{~mm}$, que es menor al de los demás grupos. Por lo que la nueva técnica propuesta presenta menor filtración apical y la técnica de cono único presentó mayor filtración.

En el gráfico 1 podemos observar la comparación de los diagramas de caja y bigotes donde encontramos que Q3 de la técnica de cono único está más alejada del cero y que el Q3 de la nueva técnica propuesta se encuentra más cercana al cero; lo cual significa que mientras las medidas son más cercanas al cero hay menor filtración. La nueva técnica presenta una pieza dentaria con una medida atípica.

\section{Discusión}

En el tratamiento de endodoncia, los factores más importantes para asegurar el éxito son: la instrumentación biomecánica del conducto radicular, la desinfección, disolución de la materia orgánica y la obturación tridimensional del espacio radicular. ${ }^{11}$

La tendencia actual del tratamiento de conductos, se ha visto revolucionada con la aparición de técnicas de ins- trumentación rotatoria que permiten estandarizar la conicidad al momento de preparar los conductos, y el uso de conos principales de gutapercha con conicidades correspondientes a cada instrumento rotatorio. Es por esto que cada vez más, aparecen nuevos y diferentes sistemas, los cuales ofrecen ser mejores que sus precedentes.

En estudios In Vitro, la utilización de diferentes tinciones como indicadores de microfiltración es común para poder evaluar las diferentes técnicas de obturación en milímetros, midiendo la penetración entre el conducto y los materiales de obturación. En el presente estudio utilizamos tinta china. Además en este estudio utilizamos el método de diafanización o transparentación, que permite identificar la tinción y poder realizar la medición.

La técnica de obturación con cono único taperizado ha generado mucha controversia en la literatura científica. Elayouti A et al. ${ }^{12}$ reportó que con la técnica de cono taperizado eran innecesarias las puntas accesorias para obturar el conducto, sin embargo Hembroug et al., ${ }^{13}$ evidencia que en conductos instrumentados con sistemas rotatorios y obturados con técnica de condensación lateral, obtuvieron mejores resultados que las piezas obturadas mediante la técnica de cono único, lo cual se corrobora en este estudio y es apoyado por otros investigadores. Ramos J. y Flórez J. ${ }^{14}$ concluyó que la técnica de condensación lateral muestra menor grado de microfiltración que la técnica de cono único, concordando con los resultados del presente estudio. Estadísticamente no se encuentra diferencias significativas. En numerosos estudios se ha demostrado que la técnica de obturación de cono único, presenta mayor microfiltración apical al compararse con la técnica de condensación lateral. ${ }^{15,16}$ Según Pommel y Camps $^{15}$ refieren que la razón es que en la técnica de cono único la gutapercha no es compactada sino introducida de acuerdo a la longitud de trabajo, permitiendo que el sellado este a cargo del cemento.

Montalván S, Meneses A y Torres J, ${ }^{17}$ no evidenciaron diferencias significativas al comparar la adaptación de los conos de gutapercha (conicidad. 02) y los conos taperizados (conicidad. 06), en el presente estudio cuando comparamos las técnicas de condensación lateral (conos. 02) y la nueva técnica propuesta (cono principal. 04 y accesorios. 02) tampoco se encontraron diferencias significativas. 
Bidar y Cols, ${ }^{18}$ no encontraron diferencias significativas entre los conductos radiculares obturados con conos de gutapercha estandarizada conicidad. 02 y conos de gutapercha conicidad. 04 empleando la técnica de obturación condensación lateral, coincidiendo con los resultados del el presente estudio.

Este estudio nos permite extrapolar los resultados en la práctica clínica, sin embargo, tiene la desventaja de que es un procedimiento In Vitro y no podemos simular las condiciones existentes en la pieza dentaria, como la ausencia de ligamento periodontal, la temperatura, humedad y $\mathrm{pH}$ al que se encuentra expuesto un conducto radicular. ${ }^{19}$ Se tiene en consideración también que los resultados están condicionados por la habilidad y práctica del operador, por lo que los resultados obtenidos pueden variar con los resultados de estudios In Vitro posteriores. Sin embargo, se sugiere abundar más el tema mediante ensayos clínicos.

\section{Conclusiones}

La nueva técnica propuesta obtuvo menor grado de microfiltración a pical en comparación con la técnica de conden-sación lateral y cono único. Sin embar-go estadísticamente no existen diferen-cias significativas entre las tres técnicas de obturación $(\mathrm{p}=0,567)$.

\section{Referencias bibliográficas}

1. Schilder H. Filling root canals in three dimensions. Dent. Clin. North. Am. 1967;11: 723-744.

2. Gutmann JL, Witherspoon DE. Sistema de obturación de los canales radiculares limpios y conformados. En: Cohen S, Burns RC, editores. Vías de la pulpa. $7^{\circ} \mathrm{ed}$. Madrid: Harcourt Mosby; 1998. p. $258-361$.

3. Sjögren U, Hägglund B, Sundqvist $\mathrm{G}$, Wing K. Factors affecting the long-term results of endodontic treatment. J. Endod. 1990; 16(10):498-504.

4. Lin LM, Skribner JE, Gaengler P. Factors associated with endodontic treatment failures. J. Endodon. 1992; 18(12):625-627.

5. Abou-Rass M, Bogen G. Microorganisms in closed periapical lesions. Int. Endod. J. 1998; 31 (1):39-47.

6. Torabinejad M, Ung B, Kettering JD. In vitro bacterial penetration of coronally unsealed endodontically treated teeth. J. Endod. 1990; 16(12):566-569.

7. Magura ME, Kafrawy AH, Brown CE, Newton CW. Human saliva coronal microleakage in obturated root canals: an in vitro study. J. Endod. 1991; 17(7):324-331.

8. Saunders WP, Saunders EM. Coronal leakage as a cause of failure in root-canal therapy: a review. Endod. Dent. Traumatol. 1994; 10(3):105-108.

9. Frajlich S, Goldberg F, Manfré S, Dreyer C. Estudio simultáneo de la capacidad de sellado apical y coronario de la obturación endodóntica. Rev. Asoc. Odontol. Argent. 1999;87(6):489-493.

10. Barzuna Mayid, Ulate Ronald. Comparación del Selle Apical de dos técnicas de obturación en endodoncia: Lateral modificada vs. Ultrasonido. Revista Dental, Universidad Latinoamericana de Ciencia y Tecnología. 2008; 1(1): $11-23$

11. Von Fraunhofer JÁ, Fagundes DK, Mc Donald NJ, Dumsha TC. The effect of root canal preparation on microleakage within endodontically treated teeth: an in vitro study. Int Endod J 2000; 33(4):355-60.
12. Elayouti A, Achleithner C, Löst C, Weiger R. Homogeneity and adaptation of a new gutta-percha paste to root canal walls. J Endod. 2005; 9: 687-90.

13. Hembrough MW, Steiman HR, Belanger KK. Lateral condensation in canals prepared with nickel titanium rotary instruments: an evaluation of the use of three different master cones. J Endod. 2002; 28(7):516-9.

14. Ramos J, Flórez J. Microfiltración apical en raíces preparadas con protaper manual y obturadas con condensación lateral y cono único. Revista Colombiana de Investigación en Odontología. 2011; 2(6): $155-162$.

15. Pommel L, Camps J. In vitro apical leakage of system B compared with other filling techniques. J En$\operatorname{dod} 2001: 27: 449-51$.

16. Yucel Ac, Ciftci A. Effects of different root canal obturation techniques on bacterial penetration. Oral Surg Oral Med Oral Pathol Oral Radiol Endod. 2006; 102: 88-92.

17. Montalván SS, Meneses A, Torres JP. Comparación microscópica de la adaptación del cono maestro de gutapercha con conicidades 2\% y $6 \%$. Rev Estomatol Herediana. 2005; 15 (2) : 107-111.

18. Bidar M, Sadeghi G, Gharechani M, Mortazavi M, Forghani M. In vitro comparison of apical leakage in root canals obturated with 0.04 and 0.02 tapered gutta-percha. Iran Endod J. 2010 Aug; 5(3): 97100 .

19. Grossman LI. Physical properties of root canal cements. J. Endod. 1976; 2:124-6. 